\title{
ASYMPTOTIC DISTRIBUTION OF SINGULARITIES OF SOLUTIONS OF MATRIX-RICCATI DIFFERENTIAL EQUATIONS
}

\author{
GERHARD JANK ${ }^{1}$
}

(Received 28 June 1990; revised 15 January 1991)

\begin{abstract}
In the present paper, we make use of the method of asymptotic integration to get estimates on those regions in the complex plane where singularities and critical points of solutions of the Matrix-Riccati differential equation with polynomial coefficients may appear. The result is that most of these points lie around a finite number of permanent critical directions. These permanent directions are defined by the coefficients of the differential equation. The number of singularities outside certain domains around the permanent critical directions, in a circle of radius $r$, is of growth $O(\log r)$. Applications of the results to periodic solutions and to the determination of critical points are given.
\end{abstract}

\section{Introduction}

Many problems in mechanics, control theory and system theory lead to a nonlinear system of differential equations of the form

$$
W^{\prime}=A(z)+B(z) W+W C(z)+W D(z) W .
$$

Here the coefficient matrices $A, B, C, D$ are matrix polynomials (in the complex variable $z$ ) of type $m \times n, m \times m, n \times n, n \times m$, respectively. According to the existence theorem (see e.g. [6]), every solution $W(z)$ of (1.1) is a locally holomorphic $m \times n$ matrix. Following W. T. Reid [14] we consider the linear system

\footnotetext{
${ }^{1}$ Lehrstuhl II für Mathematik, RWTH Aachen, Templergraben 55, D-5100 Aachen, Federal Republic of Germany.

(C) Copyright Australian Mathematical Society 1992, Serial-fee code 0334-2700/92
} 


$$
Y^{\prime}=\Omega(z) Y
$$

associated with (1.1), where

$$
\Omega=\left(\begin{array}{cc}
-C(z) & -D(z) \\
A(z) & B(z)
\end{array}\right) .
$$

As $\Omega$ is a polynomial matrix of type $(n+m) \times(n+m)$, we get from [6] that all solutions of (1.2) are entire, i.e. holomorphic in the complex plane. Let $U, V$ be matrices of type $n \times n, m \times n$ respectively, where

$$
Y=\left(\begin{array}{l}
U \\
V
\end{array}\right)
$$

is a solution of (1.2). Then it is easy to see that $W=V U^{-1}$ is a solution of (1.1) if $U^{-1}$ exists. To guarantee the existence, we may for example take solutions $Y$ of $(1.2)$ with $Y(0)=\left(\begin{array}{l}E_{n} \\ V_{0}\end{array}\right)$, where $E_{n}$ is the $n$-dimensional identity matrix and $V_{0}$ is arbitrary. On the other hand, given any solution $W$ of (1.1) with $W\left(z_{0}\right)=W_{0}, z_{0} \in \mathbf{C}$, then we take the entire solution $Y=\left(\begin{array}{l}U \\ V\end{array}\right)$ of $(1.2)$ with $Y\left(z_{0}\right)=\left(\begin{array}{c}E_{n} \\ W_{0}\end{array}\right)$. According to the uniqueness of solutions of (1.1) we get

$$
W(z)=V U^{-1} \text {. }
$$

As the right hand side is holomorphic in $\mathbf{C}$, except for a discrete set of singularities where $\operatorname{det} U=0,(1.5)$ gives a meromorphic extension of the given solution $W$. For what follows we have the important

Remark 1.1. All solutions of (1.1) are meromorphic in $\mathbf{C}$, i.e. given $z_{0} \in \mathbf{C}$, a solution $W$ has locally the Laurent representation

$$
W(z)=\sum_{k=-p}^{\infty} W_{k}\left(z-z_{0}\right)^{k}, \quad W_{k} \in \mathbf{C}^{m \times n}, p \in \mathbf{N}_{0} .
$$

For $p>0$ in (1.6) and $W_{-p} \neq 0$ we say that $W$ has a pole of order $p$ in $z_{0}$.

In the scalar case of (1.1) it is easy to see that the order of almost all poles is one (see [8, p. 219]). This is not true for the higher dimensional case, as the following example shows.

The equation

$$
\left(\begin{array}{l}
w_{1} \\
w_{2}
\end{array}\right)^{\prime}=\left(\begin{array}{l}
1 \\
0
\end{array}\right)+\left(\begin{array}{cc}
0 & -1 \\
2 & 0
\end{array}\right)\left(\begin{array}{l}
w_{1} \\
w_{2}
\end{array}\right)+\left(\begin{array}{l}
w_{1} \\
w_{2}
\end{array}\right)\left(\begin{array}{ll}
2 & 0
\end{array}\right)\left(\begin{array}{l}
w_{1} \\
w_{2}
\end{array}\right)
$$

has the solution 


$$
\left(\begin{array}{l}
w_{1} \\
w_{2}
\end{array}\right)=\left(\begin{array}{c}
\tan z \\
\tan ^{2} z
\end{array}\right)
$$

which has infinitely many poles of second order.

It is a task of this paper to get information on the asymptotic distribution of the poles of the solutions. Clearly, it depends on the coefficients in (1.1). This can be done by characterising the regions where no singularities can appear. In these regions, we can derive uniformly asymptotic estimates for the solutions. The method we use is the asymptotic integration which goes back to L. W. Thomé, H. Poincaré and G. P. Birkhoff, and was further developed by W. B. Jurkat [10], whose presentation we follow.

\section{Preliminaries}

In this section, we want to describe the tool of asymptotic integration for linear systems in the irregular singular case, and how it can be applied to the asymptotic representation of solutions of (1.1) in the neighborhood of infinity. From [10, p. 32] and [2, p. 11] we get for the system (1.2) the existence of a normalised formal fundamental system of the form

$$
H(z)=T\left(z^{1 / p}\right)\left(z^{1 / p}\right)^{L} \exp \left(Q\left(z^{1 / p}\right)\right),
$$

with a suitable $p \in \mathbf{N}$; here $T, L, Q$, denote $n+m \times n+m$ matrices. $T$ is a formal power series of the form

$$
T(t)=T_{k} t^{k}+T_{k-1} t^{k-1}+\cdots+T_{0}+T_{-1} t^{-1}+\cdots, \quad T_{j} \in \mathbf{C}^{n+m \times n+m},
$$

$L$ is a constant matrix in Jordan canonical form and $Q$ is a diagonal matrix $Q=\operatorname{diag}\left(q_{1}, \ldots, q_{n+m}\right)$, where $q_{j}, j=1, \ldots, n+m$, are polynomials with $q_{j}(0)=0$. Furthermore we have from $[2$, p. 11] that $Q L=L Q$. Notice that this condition implies that the polynomials in $Q$ which belong to one specific Jordan block of $L$ are identical.

There is an algorithm for the determination of the polynomials $q_{j}$ and the eigenvalues of $L$ up to an integer, which makes use of transformations of the system (1.2) to an ordinary differential equation of order $n+m$, with rational coefficients. This algorithm has been implemented using the computer algebra system MAPLE (cf. [3], [4]). If all polynomials $q_{1}, \ldots, q_{n+m}$ are equal, then (1.2) can easily be transformed to the regular singular case, i.e. to the case where $Q \equiv 0$ in the representation (2.1). If not all polynomials in the representation (2.1) are equal, then they define a set of Stokes directions.

Here we call $\theta$ a Stokes direction if there exist two different polynomials $q_{i}, q_{k}$ in $Q$ with the property that if

$$
q_{i}\left(z^{1 / p}\right)-q_{k}\left(z^{1 / p}\right)=z^{1 / p}\left(a_{l}+O\left(z^{-1 / p}\right)\right), \quad a_{l} \neq 0,
$$


then

$$
\lim _{|z| \rightarrow \infty, \arg z=\varphi} \frac{\mathfrak{R}\left[q_{i}\left(z^{1 / p}\right)-q_{k}\left(z^{1 / p}\right)\right]}{|z|^{1 / p}} \begin{cases}>0, & \text { if } \varphi \in(\theta, \theta+\varepsilon), \\ <0, & \text { if } \varphi \in(\theta-\varepsilon, \theta),\end{cases}
$$

for some $\varepsilon>0$. The set of all Stokes directions is discrete. If it is not empty, then we denote the Stokes directions by $\gamma_{\mu}, \mu \in \mathbf{Z}$ and arrange them so that $\gamma_{\mu}<\gamma_{m+1}$. With this we define (see [10]) the angular domains called normal sectors

$$
S_{\mu}=\left\{z \mid \gamma_{\mu-1}<\arg z<\gamma_{\mu+1}\right\}
$$

and we put $S_{\mu}=\mathrm{C}$ for all $\mu$, if there are no Stokes directions.

If we are given a solution matrix $Y$ of (1.2), then we get from [5] that to each normal sector $S_{\mu}$ as defined in (2.2) there exists a matrix $C_{\mu} \in \mathbf{C}^{(n+m) \times n}$ such that

$$
Y(z) \sim H(z) C_{\mu}
$$

is an asymptotic representation of $Y$ in $S_{\mu}$. This asymptotic relation (2.3) should be understood in the way that to each sector $S_{\mu}$ there is a fundamental solution $F_{\mu}(z)$ of (1.2) such that $F_{\mu}(z) \sim H(z)$. If we truncate the formal power series $T$ in (2.1) at a power, say $-n$, and denote the resulting finite series by $T_{n}$ then the last $\sim$ sign means

$$
F_{\mu}(z) \exp \left(-Q\left(z^{1 / p}\right)\right)\left(z^{1 / p}\right)^{-L}-T_{n}(z)=O\left(z^{-(n+1) / p}\right)
$$

as $z \rightarrow \infty$ in any closed subsector of $S_{\mu}$.

In order to define an asymptotic representation for solutions of (1.1) we divide the $n+m \times n+m$ matrix $H$ in (2.1) into two blocks $H_{1}, H_{2}$ of size $n \times n+m, m \times n+m$ respectively, such that

$$
H(z)=\left(\begin{array}{l}
H_{1}(z) \\
H_{2}(z)
\end{array}\right)
$$

Now we give

DefinItIon 2.1. The $m \times n$ matrix $\Psi(z)$ given by

$$
\Psi(z)=H_{2}(z) C_{\mu}\left(H_{1}(z) C_{\mu}\right)^{-1}
$$

is said to be an asymptotic representation of a solution $W(z)$ of (1.1) in $S_{\mu}$, if there exists a solution $Y=\left(\begin{array}{l}U \\ V\end{array}\right)$ of $(1.2)$ with $W=V U^{-1}$ and with the asymptotic representation

$$
Y \sim\left(\begin{array}{l}
H_{1} \\
H_{2}
\end{array}\right) C_{\mu}
$$

in $S_{\mu}$. Concisely we write $W \sim \Psi$.

This definition is analogous to the scalar case treated in [9]. 


\section{The distribution of poles}

Before going into a detailed description of the asymptotic distribution of the poles of a given solution $W$ of (1.1), we want to give a crude estimate on their number in a given circle of radius $r$.

Suppose that the polynomial matrix $\Omega$ in (1.3) has the form

$$
\Omega(z)=\Omega_{g} z^{g}+\Omega_{g-1} z^{g-1}+\cdots+\Omega_{0}, \quad \Omega_{j} \in \mathbf{C}^{m+n \times m+n}, j=0, \ldots, g
$$

and $\Omega_{g}$ is a nilpotent matrix of degree $k \in \mathbf{N}$. Then it follows from [8, p. 214] that all solutions of (1.2) have an order of growth less than or equal to $g+1-1 / k$. If $\Omega_{g}$ is not nilpotent, one has to put $1 / k=0$. From this it follows for the growth order $\rho$ of the determinant

$$
\rho(\operatorname{deg} U) \leq g+1-1 / k .
$$

If we denote by $N(r, W)$ and $N(r, 0, \operatorname{det} U)$ the Nevanlinna counting functions of the poles of a solution $W$ of (1.1) and of the zeros of det $U$, respectively, with $U$ from (1.5), then we have

$$
N(r, W) \leq N(r, 0, \operatorname{det} U) .
$$

Now, with the first fundamental theorem of Nevanlinna theory (cf., e.g., [13], $[8$, p. 50]) and the definition of order (see $[8$, p. 96]), the right-hand side can be estimated for a given $\varepsilon$ and for sufficiently large $r$ by

$$
N(r, \theta, \operatorname{det} U) \leq r^{g+1-1 / k+\varepsilon} .
$$

This yields together with (3.2)

$$
N(r, W) \leq r^{g+1-1 / k+e} .
$$

Denoting by $n(r, W)$ the number of poles of $W$ in the circle with radius $r$, we get from $[8, \mathbf{p} .103]$ that $N(r, W)$ and $n(r, W)$ have the same order, and hence for $\varepsilon>0$ and sufficiently large $r$ we have

$$
n(r, W) \leq r^{g+1-1 / k+\varepsilon}
$$

by (3.3).

After that crude estimation, we want to obtain estimates on the distribution of the poles. As we already pointed out, the solutions $Y$ of (1.2) are entire and the poles of solutions of (1.1) represented by (1.5) are contained in the 
set of zeros of $\operatorname{det} U$. Henceforth we will give an asymptotic estimate of $\operatorname{det} U$.

Suppose that $W$ is a solution of (1.1) and $\Psi$ its asymptotic representation in $S_{\mu}$ according to Definition 2.1. In the following, we work in one specific normal sector $S_{\mu}$ and hence for simplicity we suppress the index $\mu$. Then we get from Definition 2.1,

$$
U(z) \sim H_{1}(z) C, \quad C \in \mathbf{C}^{(m+n) \times n} .
$$

In a first step, we write the matrix elements of $H_{1}$ in the form

$$
\left(H_{1}\right)_{i j}(z)=h_{i j}(z) \exp \left(q_{j}\left(z^{1 / p}\right)\right), \quad i=1, \ldots, n, j=1, \ldots, n+m .
$$

The elements $h_{i j}$ in (3.6) are defined by (2.1) and (2.4). Then the matrix elements of $H_{1}(z) C$ are

$$
\left(H_{1}(z) C\right)_{i j}=\sum_{k=1}^{n+m} h_{i k}(z) \exp \left(q_{k}\left(z^{1 / p}\right)\right) c_{k j}, \quad i, j=1, \ldots, n,
$$

where $c_{k j}$ denotes the matrix elements of $C$.

From this we get formally

$$
\begin{aligned}
\operatorname{det}\left(H_{1} C\right) & =\sum_{\sigma \in S_{n}} \operatorname{sign}(\sigma) \prod_{j=1}^{n}\left(H_{1}(z) C\right)_{\sigma(j), j} \\
& =\sum_{\sigma \in S_{n}} \operatorname{sign}(\sigma) \prod_{j=1}^{n}\left(\sum_{k=1}^{n+m} h_{\sigma(j), k}(z) c_{k, j} \exp \left(q_{k}\left(z^{1 / p}\right)\right)\right),
\end{aligned}
$$

where $\sigma$ denotes a permutation of $1, \ldots, n$. To study this determinant asymptotically, later on we have to truncate all the formal power series in the formal expressions $h_{\sigma(j), k}(z)$. Before doing this we define the set $M(Q)$ as the set of all polynomials $q$ of the form

$$
q=\sum_{\nu=1}^{l} \delta_{\nu} q_{j_{\nu}}, \quad \delta_{\nu}=0,1,
$$

where $q_{j_{v}}$ denotes the pairwise distinct polynomials from the matrix $Q$ in (2.1). Herewith the determinant in (3.8) can be written as

$$
\operatorname{det}\left(H_{1} C\right)=\sum_{p_{k} \in M(Q)} h_{k}(z) \exp \left(p_{k}\left(z^{1 / p}\right)\right),
$$

where the nonvanishing expressions $h_{k}$ are sums of products of the expressions $h_{\sigma(j), k}(z)$ in (3.8). 
To the set of Stokes directions of the polynomials in $Q$, denoted by $\gamma_{\mu}$, there is now an additional set of Stokes directions defined by the polynomials in $M(Q)$. This set can be ordered as before. Assume now that $\alpha$ is such a Stokes direction with

$$
\gamma_{\mu-1}<\alpha<\gamma_{\mu+1}
$$

and $\alpha_{-}$its predecessor with

$$
\gamma_{\mu-1} \leq \alpha_{-}<\alpha<\gamma_{\mu+1} .
$$

Then we say a polynomial $p_{j}$ in (3.9) is leading in the angular domain

$$
S_{\varepsilon}^{-}=\left\{z \mid \alpha_{-}+\varepsilon<\arg z<\alpha\right\}, \quad \varepsilon \geq 0
$$

if $h_{j} \neq 0$, and if, for some $\vartheta \in\left(\alpha_{-}, \alpha\right)$ with $z=r e^{i \theta}$ and for all polynomials $p_{k}$ in (3.9) with $h_{k} \neq 0$, the quotient

$$
\frac{\exp \left(p_{k}\left(r^{1 / p} e^{i \theta / p}\right)\right)}{\exp \left(p_{j}\left(r^{1 / p} e^{i \theta / p}\right)\right)}
$$

tends to zero for $k \neq j$ as $r \rightarrow \infty$.

Notice now that if $k \neq j$, and if

$$
p_{k}\left(z^{1 / p}\right)-p_{j}\left(z^{1 / p}\right)=a z^{b}(1+o(1)), \quad z \rightarrow \infty,
$$

we have

$$
\lim _{|z| \rightarrow \infty, \alpha_{-}<\arg z<\alpha} \frac{\mathfrak{R}\left[p_{k}\left(z^{1 / p}\right)-p_{j}\left(z^{1 / p}\right)\right]}{|z|^{b}}<0 .
$$

Assuming now that in (3.9) the polynomial $p_{j}$ is leading in $S_{\varepsilon}^{-}$, then we write formally

$$
\operatorname{det}\left(H_{1} C\right)=\exp \left(p_{j}\left(z^{1 / p}\right)\right)\left(h_{j}(z)+g_{j}(z)\right)
$$

with

$$
g_{j}(z)=\sum_{p_{k} \in M(Q), k \neq j} h_{k}(z) \exp \left(p_{k}\left(z^{1 / p}\right)-p_{j}\left(z^{1 / p}\right)\right) .
$$

In order to get a useful asymptotic estimate for $\operatorname{det} U$, with $U$ from (3.5), we need information about the asymptotic behavior of $g_{j}(z), h_{j}(z)$ and $h_{k}(z)$, respectively. If $\lambda_{j}$ is an eigenvalue of multiplicity $m_{j}$ of the matrix $L$ in (2.1), then we get together with (2.1), (2.4) and (3.6)

$$
h_{i j}(z)=z^{\lambda_{j} / p} P_{i j}(\log z),
$$

where $P_{i j}$ denotes a polynomial with formal power series as coefficients. It has the maximal degree $m_{j}-1$. 
Analogously to the definition of the set polynomials $M(Q)$, we now define the set $M(L)$ as the set of the complex numbers

$$
\lambda=\sum_{k=1}^{l} \delta_{k} \lambda_{k} / p, \quad \delta_{k}=0,1,
$$

where $\lambda_{k}$ denotes the eigenvalues of $L$ in (2.1).

Herewith, together with (3.9) and (3.13), we can write the formal expression $h_{k}(z)$ as

$$
h_{k}(z)=\sum_{\lambda_{j} \in M(L)} z^{\lambda_{j}} P_{j}(\log z),
$$

where the $P_{j}$ 's denote polynomials with formal power series as coefficients. If we truncate this formal series at any power and denote the resulting functions by $P_{j}^{*}(\log z)$, then there exists $K>0$ such that for sufficiently large $|z|$ we have

$$
\left|h_{k}^{*}(z)\right|=\left|\sum_{\lambda_{j} \in M(L)} z^{\lambda_{j}} P_{j}^{*}(\log z)\right|<|z|^{K} ;
$$

hence we have together with (3.12)

$$
\left|g_{j}^{*}(z)\right| \leq \sum_{p_{k} \in M(Q), i \neq j, h_{k} \neq 0}|z|^{K} \exp \left(\Re\left[p_{k}\left(z^{1 / p}\right)-p_{j}\left(z^{1 / p}\right)\right]\right) .
$$

Here once again $g_{j}^{*}(z)$ denotes the resulting function after truncating the formal series in the formal expressions $g_{j}(z)$ in (3.12).

In the same way as in [9] we can now estimate the right-hand side in (3.16).

Lemma 3.1. If $\alpha$ is a Stokes direction of the polynomials $p_{j}$ and $p_{k}$, with nonvanishing $h_{j}$ and $h_{k}$, respectively, in (3.16) and leading $p_{j}$ in $S_{\varepsilon}^{-}$, then there exists $\delta>0$ such that

$$
\sum_{p_{k} \in M(Q), k \neq j, h_{k} \neq 0}|z|^{K} \exp \left(\mathfrak{R}\left[p_{k}\left(z^{1 / p}\right)-p_{j}\left(z^{1 / p}\right)\right]\right)=o\left(\exp \left(-|z|^{\delta}\right)\right),
$$

as $z \rightarrow \infty$ in $S_{\varepsilon}^{-} \backslash \Lambda(\alpha)$, where $\Lambda$ is defined by

$$
\Lambda(\alpha)=\left\{z=r e^{i \theta}|| \theta-\alpha \mid \leq r^{-1 / 2 p} \log r\right\},
$$

and $\varepsilon \in\left(0, \alpha-\alpha_{-}\right)$.

Proof. Put for $j$ fixed $\rho_{k}=\frac{1}{p} \operatorname{deg}\left(p_{j}-p_{k}\right), \rho=\max _{h_{k} \neq 0} \rho_{k}$ and $p_{j}\left(z^{1 / p}\right)-$ $p_{k}\left(z^{1 / p}\right)=d_{k} z^{\rho_{k}}+\cdots$ (lower order terms). Herewith we define

$$
\psi_{k}(\theta)=\Re\left(d_{k} e^{i \rho_{k} \theta}\right)
$$


Then there exists $A>0$, such that for sufficiently large $|z|=r$ and for all $k$ in (3.16) with $h_{k} \neq 0$

$$
\left|p_{k}\left(z^{1 / p}\right)-p_{j}\left(z^{1 / p}\right)-d_{k} z^{\rho_{k}}\right| \leq A r^{\rho-1 / p} .
$$

If

$$
2 \beta=\min _{k, h_{k} \neq 0}\left|\psi_{k}^{\prime}(\alpha)\right|,
$$

then $2 \beta>0$, because $p_{j}$ is leading and there are no zeros of the functions $\psi_{k}(\theta)$ in $\left(\alpha_{-}, \alpha\right)$. Otherwise we would have a further Stokes direction in this interval. From $\psi_{k}(\theta)=\psi_{k}(\alpha)+\psi_{k}^{\prime}(\alpha)(\theta-\alpha)+o(\theta-\alpha)$, with $\psi_{k}(\alpha) \geq 0$, we get for sufficiently large $|z|=r$ outside $\Lambda(\alpha)$, the estimate

$$
\psi_{k}(\theta)>\beta r^{-1 / 2 p} \log r \text {. }
$$

All these properties are easily to be seen if one keeps in mind that the functions $\psi_{j}$ and $\psi_{k}$ are "cosine-lines". Hence together with (3.18) we get with $\delta=1 / 4 p$

$$
\begin{aligned}
& |z|^{K} \exp \left(\Re\left[p_{k}\left(z^{1 / p}\right)-p_{j}\left(z^{1 / p}\right)\right]\right) \\
& \quad \leq \exp \left(-\beta r^{\rho-1 / 2 p} \log r+A r^{\rho-1 / p}+K \log r\right)=o\left(\exp \left(-r^{\delta}\right)\right)
\end{aligned}
$$

for sufficiently large $|z|=r$, outside $\Lambda(\alpha)$. This proves the lemma since $\beta$ is independent of $k$ and together with (3.16) we have

$$
\left|g_{j}^{*}(z)\right|=o\left(\exp \left(-|z|^{\delta}\right)\right), \quad z \rightarrow \infty,
$$

in $S_{\varepsilon}^{-} \backslash \Lambda(\alpha)$

Definition 3.1. Let $W$ be a solution of (1.1) in the form $W=V U^{-1} U \sim$ $H_{1} C_{\mu}$ in a normal sector $S_{\mu}$. The set of polynomials

$$
C(W)=\bigcup_{\mu \in \mathbf{Z}}\left\{p_{k} \in M(Q) \mid h_{k} \neq 0 \text { in (3.9) }\right\}
$$

defines a subset of Stokes directions of the set of Stokes directions belonging to $M(Q)$. A Stokes direction which is defined by the polynomials $p_{k} \in C(W)$ is called a permanent critical direction of $W$; the half ray through the origin in this direction is called a permanent critical line of $W$.

With respect to the distribution of poles of a solution of (1.1) we have now

THEOREM 3.1. The poles of a solution $W$ of (1.1) are-except for a number $n_{1}(r, W) \leq O(\log r)$-located in a finite number of domains $\Lambda$, defined in 
(3.17), around the permanent critical lines of $W$. Here with $n_{1}(r, W)$ we denote the number of poles of $W$ in the circle of radius $r$ and outside all the domains $\Lambda$ around the permanent critical directions.

Notice that the permanent critical directions can be computed from the coefficient matrices $A, B, C, D$ of (1.1), by computing the Stokes directions of the formal solution in (2.1). These directions do not depend on the initial value of the regarded solution $W$.

Proof. Suppose that $W$ is a solution (1.1) given as $W=V U^{-1}$ which, according to Definition 2.1 , has an asymptotic representation in a normal sector $S_{\mu}$ with the matrix $C_{\mu}=C$.

In the first step we derive the formal expression (3.12) and an asymptotic estimate of the "remaining term" (3.16). In the second step we have to find an asymptotic estimate for the function $h_{j}^{*}(z)$ defined by the formal expression $h_{j}(z)$ in (3.12) after truncating the formal series at certain powers. From (3.14) we see that $h_{j}$ can be written as

$$
h_{j}(z)=\sum_{\lambda_{\nu} \in M(L)} z^{\lambda_{\nu}+\kappa_{\nu}}\left(Q_{\nu}(\log z)+R_{\nu}(\log z)\right),
$$

where the polynomials $Q_{\nu}$ have constant coefficients and the coefficients of the polynomials $R_{\nu} \neq 0$ are still formal series, but all of them are starting with a negative power of $z^{1 / p}$. If $Q_{\nu}=0$, then $R_{\nu}=0$.

To get asymptotic estimates, we have to truncate the formal series in $R_{\nu}$. We take only the first nonvanishing term of each series and denote the remainder by $R_{\nu}^{*}(\log z)$. Since by construction all coefficients of this function contain a negative power of $z$, we get

$$
R_{\nu}^{*}(\log z)=o(1), \quad z \rightarrow \infty .
$$

This together with (3.22) gives

$$
h_{j}^{*}(z)=\sum_{\lambda_{\nu} \in M(L), Q_{\nu} \neq 0} z^{\lambda_{\nu}+\kappa_{\nu}}\left(Q_{\nu}(\log z)+o(1)\right) .
$$

If we denote by $\kappa$ the maximum of $\mathfrak{R}\left(\lambda_{\nu}+\kappa_{\nu}\right)$ in (3.24), then with $\tau_{\nu}=\mathfrak{I} \lambda_{\nu}$ we get from (3.24)

$$
h_{j}^{*}(z)=z^{\kappa} \sum_{\mathfrak{R}\left(\lambda_{\nu}+\kappa_{\nu}\right)=\kappa, Q_{\nu} \neq 0} z^{i \tau_{\nu}}\left(Q_{\nu}(\log z)+o(1)\right) .
$$

Here we used the boundedness of the terms $z^{i \tau_{\nu}}$ in an angular domain. If we now denote by $s$ the maximum degree of the polynomials $Q_{\nu}$ in (3.25), then we can write (3.25) as

$$
h_{j}^{*}(z)=z^{\kappa}(\log z)^{s}\left[\sum_{\nu} c_{\nu} z^{i \tau_{\nu}}+o(1)\right],
$$


where the sum has to be taken over all $\nu$ with $\mathfrak{R}\left(\lambda_{\nu}+\kappa_{\nu}\right)=\kappa, Q_{\nu} \neq 0$, $\operatorname{deg} Q_{\nu}=s$. The function

$$
f(z)=\sum_{\nu} c_{\nu} z^{i \tau_{\nu}}
$$

in (3.26) changes, with the usual transformation $z=e^{u}$, into an exponential sum

$$
g(u)=f\left(e^{u}\right)=\sum_{\nu} c_{\nu} e^{i u \tau_{\nu}}
$$

The order of $g$ is one and its type is finite; hence by the first fundamental theorem of Nevanlinna theory (cf., e.g., [8] p. $47 \mathrm{ff}$. and p. 103 Theorem 11.4), we get for the number of zeros of $g$ in a circle of radius $R$

$$
n(R, 0 ; g)=O(R), \quad R \rightarrow \infty,
$$

and hence with $R=\log r+o(1)$

$$
n(r, 0 ; f)=O(\log r)
$$

From $[12$, p. 267] we get from a theorem concerning almost periodic functions such as any finite exponential sum $g$ that for any $\delta>0$ there exists $m(\delta)>0$, such that

$$
|g(u)| \geq m(\delta)
$$

in a strip $|\mathfrak{I} u| \leq h$ and outside the circles of radius $\delta$ around the zeros $u_{k}$ of $g$. This gives for $f$ in (3.27) the estimate

$$
|f(z)| \geq m(\delta)
$$

outside the sets $B_{k}(\delta)=\left\{z|| \log z-u_{k} \mid \leq \delta\right\}$.

If we apply Rouché's theorem (cf., e.g., Ahlfors [1], p. 153) to (3.26) and $f$ in (3.27), then we get together with (3.27) that $h_{j}^{*}(z)$ in (3.26) and $f$ have the same number of zeros in the sets $B_{k}(\delta)$. From (3.5), (3.12) together with (3.21), (3.26) and (3.27) we have

$$
\begin{aligned}
\operatorname{det} U & =\exp \left(p_{j}\left(z^{1 / p}\right)\right)\left(h_{j}^{*}+g_{j}^{*}\right) \\
& =\exp \left(p_{j}\left(z^{1 / p}\right)\right)\left[z^{\kappa}(\log z)^{s}(f(z)+o(1))+o\left(e^{-|z|^{\delta}}\right)\right] \\
& =\exp \left(p_{j}\left(z^{1 / p}\right)\right) z^{\kappa}(\log z)^{s}(f(z)+o(1)), \quad z \rightarrow \infty, \quad z \in S_{\varepsilon}^{-} \backslash \Lambda .
\end{aligned}
$$

This, together with (3.29) and (3.30), gives the desired result of Theorem 3.1, since we have at most a finite number of sectors $S_{e}^{-}$, and we get analogous estimates in the corresponding finite number of sectors $S_{e}^{+}=\{z \mid \alpha<\arg z<$ $\left.\alpha_{+}-\varepsilon\right\}$, where $\alpha_{+}$denotes the Stokes direction following $\alpha$.

It is quite clear from the proof that the existence of an infinite number of poles, outside the domains $\Lambda$ around the permanent critical lines depends on the existence of an infinite number of zeros of the sum in (3.26). In general, this depends on the matrix $C$ in (3.5), and hence on the initial value of the specific solution $W$ of (1.1). In some special situations, however, the sum in 
(3.26) has at most a finite number of zeros in any domain $S_{\varepsilon}^{-} \backslash \Lambda$ and $S_{\varepsilon}^{+} \backslash \Lambda$. If, for example, all the eigenvalues $\lambda_{l}$ of the matrix $L$ in the formal solution (2.1) are real, then its imaginary parts $\tau_{l}$ are zero and the corresponding sum $f$ in (3.27) reduces to a constant.

\section{Growth estimates and critical points}

If we use the matrix norm

$$
\|W\|=\sum_{j=1}^{n} \sum_{i=1}^{m}\left|w_{i j}\right|
$$

for any solution of (1.1), then we get a general estimate of $\|W\|$ outside the critical domains.

THEOREM 4.1. Suppose that $W$ is a solution of (1.1) and

$$
\rho=(1 / p) \max _{j=1, \ldots, n+m} \operatorname{deg} q_{j},
$$

where $q_{j}$ denotes the polynomials in the matrix $Q$ in (2.1). Then for some constant $K>0$ and sufficiently large $|z|=r$ we have

$$
\log ^{+}\|W(z)\| \leq K\left(r^{\rho}+\log r\right)
$$

for $z$ outside the domains $\Lambda$ around the permanent critical directions and outside the domains $B_{k}(\delta)$ defined in (3.30).

Proof. Once again we start with the representation $W=V U^{-1}$. The matrix elements $v_{i j}$ of $V$ have, according to Definition 2.1, an asymptotic representation

$$
\begin{aligned}
v_{i j} & =\left(H_{2}(z) C_{\mu}\right)_{i j} \\
& =\sum_{k=1}^{n+m} h_{n+i, k}(z) \exp \left(q_{k}\left(z^{1 / p}\right)\right) c_{k j}, \quad i=1, \ldots, m, j=1, \ldots, n
\end{aligned}
$$

in a normal sector $S_{\mu}$. This leads in any normal sector immediately to

$$
\left|v_{i j}(z)\right| \leq|z|^{K} e^{\tau|z| \rho},
$$

for some constants $K, \tau>0$ and for sufficiently large $|z|$. Now let the matrix elements $\alpha_{i j}$ of $U^{-1}$ be represented as

$$
\alpha_{i j}=U_{j i} / \operatorname{det} U \text {, }
$$


where $U_{j i}$ denotes the adjoint elements to $u_{j i}$ of the matrix $U$. From (3.31) we get

$$
|\operatorname{det} U|=\exp \left(\Re p_{j}\left(z^{1 / p}\right)\right)|z|^{\kappa}|\log z|^{s}|f(z)+o(1)|, \quad z \rightarrow \infty, \quad z \in S_{\varepsilon}^{-} \backslash \Lambda .
$$

From (3.30) we have $|f(z)| \geq m(\delta)$ and hence for sufficiently large $|z|$,

$$
|f(z)+o(1)| \geq \frac{1}{2} m(\delta)
$$

outside the domains $B_{k}(\delta)$. This gives

$$
|\operatorname{det} U(z)| \geq \exp \left(\Re p_{j}\left(z^{1 / p}\right)\right)|z|^{\kappa}|\log z|^{s} \frac{m(\delta)}{2},
$$

for sufficiently large $z$ in $S_{\varepsilon}^{-} \backslash \Lambda$ and outside $B_{k}(\delta)$.

Since the adjoint elements $U_{j i}$ are determinants of elements of $U$ we get for each of them an analogous representation like (4.6). If we notice now that any function $f$ is bounded in an angular domain, say by a constant $c>0$, then we get for sufficiently large $z$ in $S_{\varepsilon}^{-} \backslash \Lambda$ and some $p \in M(Q), \tilde{\kappa}$ and $\tilde{s}$

$$
\left|U_{j i}(z)\right| \leq 2 c \exp \left(\mathfrak{R} p\left(z^{1 / p}\right)\right)|z|^{\tilde{\kappa}}|\log z|^{\tilde{s}} .
$$

The estimates (4.7) and (4.8), together with (4.5), then give

$$
\left|\alpha_{i j}\right| \leq|z|^{K} e^{\tau|z|^{\rho}}
$$

for suitable constants $K, \tau>0$ and for sufficiently large $|z|$ with $z$ outside the sets $\Lambda$ around the permanent critical directions and outside the sets $B_{k}(\delta)$. Now (4.4) and (4.9), together with (1.5) and (4.1), give (4.2). Estimates of this type for differential equations with constant coefficients are given in [11].

Notice that the exceptional domains $B_{k}(\delta)$ can be omitted in Theorem 4.1 if we are dealing with a differential equation (1.1) having for example only real eigenvalues in the matrix $L$ of (2.1). Next we want to get information on the critical point of (1.1), i.e. on the zeros of $W^{\prime}$.

THEOREM 4.2. Let a differential equation (1.1) be given and $Q$ the associated matrix in the formal fundamental system (2.1), $q_{j_{i}}, i=1, \ldots, l$, the pairwise distinct polynomials in $Q$. Define the set of polynomials $M_{1}(Q)$ as

$$
M_{1}(Q)=\left\{q \mid q=\sum_{i=1}^{l} \alpha_{i} q_{j_{i}}, \alpha_{i}=0, \pm 1, \alpha_{1}^{2}+\cdots+\alpha_{l}^{2} \neq 0\right\} .
$$

Then the critical points of any solution $W$ (i.e. the points with $W^{\prime}=0$ ) areexcept for $O(\log r)$ many-located in the union of a finite number of domains 
$\Lambda$, defined in (3.17), around the Stokes directions defined by the polynomials in $M_{1}(Q)$, and the sets $B_{k}(\delta)$ as defined by (3.30).

Proof. Analogously to the proof of Theorem 3.1. we get from (4.3), if $\tilde{q}_{j}$ is thought to be leading, with appropriate constants $\kappa_{\nu j}, s_{\nu j}$

$$
\begin{aligned}
v_{\nu j}(z) & =\exp \left(\tilde{q}_{j}\left(z^{1 / p}\right)\right)\left\{\sum_{l} h_{n+\nu, l}^{*} c_{l j}+o\left(e^{-|z|^{\delta}}\right)\right\} \\
& =\text { const. } \exp \left(\tilde{q}_{j}\left(z^{1 / p}\right)\right) z^{\kappa_{\nu j}}(\log z)^{s_{\nu j}}\left(f_{\nu j}+o(1)\right), \quad z \rightarrow \infty, \quad z \in S_{\varepsilon}^{-} \backslash \Lambda,
\end{aligned}
$$

where $f_{\nu j}$ denotes a function as in (3.27). From (3.31) we now get the determinant of $U$ and, as the adjoint elements in (4.5) are of the same type, we get a quotient of functions of type (3.31) as an asymptotic representation of $\alpha_{i j}$ in (4.5). Together with (1.5), if we denote the elements of $W$ by $w_{\nu j}$, we see that there exists a polynomial $q_{\nu j} \in M_{1}(Q)$, leading in $S_{\varepsilon}^{-} \backslash \lambda$ and constants $\kappa_{\nu j}, s_{\nu j}$ such that

$$
w_{\nu j}(z)=\sum_{l=1}^{n+m} v_{\nu l} \alpha_{l j}=\exp \left(q_{\nu j}\left(z^{1 / p}\right)\right) z^{\kappa_{\nu j}}(\log z)^{s_{\nu j}} \frac{f_{\nu j}(z)+o(1)}{f(z)+o(1)}, \quad z \rightarrow \infty,
$$

where $f$ and $f_{\nu j}$ are functions of type (3.27), respectively. Since we are dealing with the polynomials $q \in M_{1}(Q)$ we have to partition the normal sectors (defined by the polynomials $Q$ ) by introducing additional Stokes directions defined by the polynomials in $M_{1}(Q)$. Now $S_{\varepsilon}^{-}$in (4.10), (4.11) has to be defined with respect to this new set of Stokes directions.

Differentiating in (4.11) and collecting the resulting terms yields in the case of $\operatorname{deg} q_{\nu j} \leq p$

$$
\begin{aligned}
w_{\nu j}^{\prime}(z)=\exp \left(q_{\nu j}\left(z^{1 / p}\right)\right) z^{\kappa_{\nu j}}(\log z)^{s_{\nu j}} \frac{g_{\nu j}(z)+o(1)}{f^{2}(z)+o(1)}, & \\
& z \rightarrow \infty, \quad z \in S_{\varepsilon}^{-} \backslash \Lambda, z \notin B_{k}(\delta),
\end{aligned}
$$

where $g_{\nu j}$ denotes some new function of the type as defined in (3.27). If in (4.11) $m=\operatorname{deg} q_{\nu j}>p$, then we get analogously

$$
\begin{aligned}
w_{\nu j}^{\prime}(z)=\exp \left(q_{\nu j}\left(z^{1 / p}\right)\right) z^{\kappa_{\nu j}+(m-p) / p} & (\log z)^{s_{\nu j}} \frac{f_{\nu j}(z)+o(1)}{f(z)+o(1)}, \\
z & \rightarrow \infty, \quad z \in S_{\varepsilon}^{-} \backslash \Lambda, \quad z \notin B_{k}(\delta) .
\end{aligned}
$$

From (4.12) and (4.13), together (3.30) it follows that-except for a finite number-the zeros of $W^{\prime}$ which are not in the domain $S_{\varepsilon}^{-} \backslash \Lambda$, and not in 
the domains $B_{k}(\delta)$ as defined in (3.30), have to be zeros of the functions $g_{\nu j}+o(1)$ or $f_{\nu j}+o(1)$. Since these functions are once again of the type as defined in (3.27), we get analogously to the proof of Theorem 3.1 that there are at most $O(\log r)$ such zeros. Since we have at most a finite number of sectors $S_{e}^{-}$and at most a finite number of sectors $S_{\varepsilon}^{+}$, where we get analogous estimates, the theorem is proved.

\section{Periodic solutions}

In this section, we will study how asymptotic methods can be used to get results on the possible periodicity of solutions not only of equations with polynomial coefficients but even on Matrix-Riccati equations with certain periodic coefficients. The existence of periodic positive semidefinite solutions of a Matrix-Riccati equation with periodic coefficients is studied in [7].

We start with an example of an equation of type (1.1) with nonconstant polynomial coefficients, having a periodic solution.

The differential equation

$$
\left(w_{1}, w_{2}\right)^{\prime}=2\left(w_{1}, w_{2}\right)-\left(w_{1}, w_{2}\right)\left(\begin{array}{cc}
1 & 0 \\
0 & 2 z
\end{array}\right)-\left(w_{1}, w_{2}\right)\left(\begin{array}{c}
0 \\
4(z-1)
\end{array}\right)\left(w_{1}, w_{2}\right)
$$

has the periodic solution

$$
\left(w_{1}, w_{2}\right)=\left(e^{z}, 0\right)
$$

In general the solutions of a Matrix-Riccati equation will not be entire. For periodic solutions of (1.1) with poles we have

THEOREM 5.1. Let $W$ be a nonentire periodic solution of (1.1) with period $\omega \in \mathbf{C} \backslash\{0\}$.

Then $\arg \omega=\gamma_{\mu}$, where $\gamma_{\mu}$ is one of the Stokes directions defined in the preliminary part.

Proof. If $\omega$ is a period and $z_{0}$ a pole of a given solution $W$ of (1.1), then $W$ has poles in $z_{0}+k \omega, k \in \mathbf{Z}$. Now define a small angular domain $V_{\varepsilon}=\{z \in \mathbf{C} \mid \arg \omega-\varepsilon<\arg z<\arg \omega+\varepsilon\}$ and denote by $n_{\varepsilon}(r, W)$ the number of poles of $W$ in $V_{\varepsilon} \cap\{z|| z \mid<r\}$. Then there exists $k_{0} \in \mathbf{N}$, such that for all $k>k_{0}$ the poles $z_{0}+k w$ are in $V_{\varepsilon}$ and hence there exists a constant $K>0$ such that

$$
n_{e}(r, W) \geq K r .
$$

Assume that $\arg \omega \neq \gamma_{\mu}$ for all $\mu \in \mathbf{Z}$. Then $V_{e} \cap \Lambda\left(\gamma_{\mu}\right), \Lambda\left(\gamma_{\mu}\right)$ defined in (3.17), is empty or bounded for all $\mu$ and hence contains at most a finite 
number of poles of $W$. On the other hand, it follows from Theorem 3.1. that the number of poles in $|z|<r$ and $V_{\varepsilon} \backslash \Lambda\left(\gamma_{\mu}\right)$ is at most $O(\log r)$. This contradicts (5.1).

If a periodic solution $W$ has no poles, there is one specific situation where Theorem 5.1. still can be applied.

REMARK 5.1. If $W$ is a nonconstant periodic entire solution of (1.1) with period $\omega$, and if $W$ is a matrix of type $n \times n$, then

$$
\arg \omega=\gamma_{\mu}, \quad \mu \in \mathbf{Z},
$$

where $\gamma_{\mu}$ denotes the Stokes directions for the equation $Y^{\prime}=\Omega_{1} Y$ with

$$
\Omega_{1}=\left(\begin{array}{cc}
B+W_{0} D & A+B W_{0}+W_{0} C+W_{0} D W_{0} \\
-D & -C-D W_{0}
\end{array}\right)
$$

and $W_{0}=W\left(z_{0}\right)$ for some $z_{0} \in \mathbf{C}$.

Proof. The matrix function $V=\left(W-W_{0}\right)^{-1}$ is $\omega$-periodic with poles and is a solution of

$V^{\prime}=-D-\left(C+D W_{0}\right) V-V\left(B+W_{0} D\right)-V\left(A+B W_{0}+W_{0} C+W_{0} D W_{0}\right) V$,

because $V^{\prime}=-V W^{\prime} V$, which is of type (1.1). Using (1.3) we get (5.2).

Finally we want to point out that this result can be applied to a specific class of Matrix-Riccati differential equation with periodic coefficients. Though the theory of asymptotic integration would yield results for a larger class of coefficients (i.e. for rational coefficients), we want to stay here within the framework of this paper, that means to ensure that (1.1) has globally meromorphic solutions.

Suppose the coefficients of (1.1) to be of the form:

$$
\begin{array}{ll}
A(z)=\sum_{j=1}^{n_{1}} A_{j}\left(\exp \left(\frac{2 \pi i}{\omega} z\right)\right)^{j}, & B(z)=\sum_{j=1}^{n_{2}} B_{j}\left(\exp \left(\frac{2 \pi i}{\omega} z\right)\right)^{j}, \\
C(z)=\sum_{j=1}^{n_{3}} C_{j}\left(\exp \left(\frac{2 \pi i}{\omega} z\right)\right)^{j}, & D(z)=\sum_{j=1}^{n_{4}} D_{j}\left(\exp \left(\frac{2 \pi i}{\omega} z\right)\right)^{j},
\end{array}
$$

where $A_{j}, B_{j}, C_{j}, D_{j}$ are constant matrices.

With the transformation $\zeta=\exp \left(\frac{2 \pi i}{\omega} z\right)$ we get that

$$
\begin{array}{lll}
\tilde{A}(\zeta)=A\left(\frac{\omega}{2 \pi i} \log \zeta\right), & \widetilde{B}(\zeta)=B\left(\frac{\omega}{2 \pi i} \log \zeta\right), \\
\tilde{C}(\zeta)=C\left(\frac{\omega}{2 \pi i} \log \zeta\right), & \widetilde{D}(\zeta)=D\left(\frac{\omega}{2 \pi i} \log \zeta\right)
\end{array}
$$

are polynomials with a zero in 0 . 
If we also transform the differential equation (1.1) and (1.2) accordingly, we get from (1.2)

$$
\frac{2 \pi i}{\omega} \zeta \widetilde{Y}^{\prime}=\widetilde{\Omega}(\zeta) \widetilde{Y}
$$

with

$$
\widetilde{\Omega}=\left(\begin{array}{cc}
-\tilde{C}(\zeta) & -\widetilde{D}(\zeta) \\
\tilde{A}(\zeta) & \widetilde{B}(\zeta)
\end{array}\right)
$$

Since $\frac{1}{\zeta} \widetilde{\Omega}(\zeta)$ is still a polynomial matrix, our previous theory applies in that case.

If the complex variable $z$ is considered to be real, the treated nonautonomous differential equation (1.1) occurs for example in linear-quadratic control problems or in Nash-games. It turns out that the asymptotic behavior of the solutions will be quite different depending on whether the positive real axis points in a Stokes direction of (1.1) or not. Furthermore in the latter case, the eigenvalues of the matrix $L$ in (2.1) can be used to decide if there is a uniformly asymptotic behavior of a solution or not. The influence of the initial data on the asymptotic behavior of solutions is not studied in this paper.

\section{Examples}

To illustrate the results of the preceding parts, we shall give two examples. The first example shows that there are differential equations which may have solutions with an infinite number of poles, located outside the domains $\Lambda$ around the permanent Stokes directions. Their number must be of growth $O(\log r)$. The second example shows that there exist equations with solutions which have at most a finite number of poles outside these regions.

The necessary computational effort is-except for some trivial casesrelatively high; hence we use a program package (implemented within the computer algebra system MAPLE). This package, called ELISE, is described in [4] and implemented on a 68020-processor based UNIX workstation.

1. EXAMPLE. The equation

$$
\begin{aligned}
\left(\begin{array}{l}
w_{1} \\
w_{2}
\end{array}\right)^{\prime}= & -\left(\begin{array}{c}
0 \\
4 z^{2}+2 z+9
\end{array}\right)-\left(\begin{array}{cc}
0 & 1 \\
4 z^{2}+z+12 & z^{2}+6
\end{array}\right)\left(\begin{array}{l}
w_{1} \\
w_{2}
\end{array}\right) \\
& -\left(\begin{array}{l}
w_{1} \\
w_{2}
\end{array}\right)\left(\begin{array}{ll}
1 & 0
\end{array}\right)\left(\begin{array}{l}
w_{1} \\
w_{2}
\end{array}\right)
\end{aligned}
$$


leads together with (1.2) and (1.3) to the linear system

$$
\left(\begin{array}{c}
u \\
v_{1} \\
v_{2}
\end{array}\right)^{\prime}=\left(\begin{array}{ccc}
0 & 1 & 0 \\
0 & 0 & 1 \\
-4 z^{2}-2 z-9 & -4 z^{2}-z-12 & -z^{2}-6
\end{array}\right)\left(\begin{array}{c}
u \\
v_{1} \\
v_{2}
\end{array}\right)
$$

and the solutions can be written as

$$
\left(\begin{array}{l}
w_{1} \\
w_{2}
\end{array}\right)=\frac{1}{u}\left(\begin{array}{l}
v_{1} \\
v_{2}
\end{array}\right)
$$

Now ELISE gives the following expressions for the formal fundamental system in (2.1):

$$
\exp Q\left(z^{1 / p}\right)=\exp \left(\left(\begin{array}{ccc}
-\frac{1}{3} z^{3}-2 z & 0 & 0 \\
0 & -2 z & 0 \\
0 & 0 & -2 z
\end{array}\right)\right)
$$

and for the eigenvalues of $L(\bmod$ an integer),

$$
\lambda_{1}=-3, \quad \lambda_{2}=-i, \quad \lambda_{3}=i .
$$

The Stokes directions are $\pi / 6, \pi / 2(\bmod 2 \pi / 3)$. The CPU-time used from ELISE was 9 seconds. According to Theorem 3.1., the poles of solutions are, except for a number of $O(\log r)$, in domains $\Lambda$ around that 6 directions.

Note that, together with the asymptotic existence theorem, there exist solutions $\left(u, v_{1}, v-2\right)^{\top}$ with an asymptotic representation such that

$$
u(z)=(1+o(1)) e^{-2 z} z^{k}\left(c_{1} z^{i}+c_{2} z^{-i}\right), \quad c_{1}, c_{2} \in \mathbf{C} .
$$

This shows that there are solutions of the Matrix-Riccati equation with infinitely many poles outside the domains $\Lambda$ around the critical lines. The location of this $O(\log r)$ many poles depends on the constants $c_{1}, c_{2}$, i.e. on the initial value of the solution.

EXAMPLE 2. Here we start with an equation

$$
\begin{aligned}
\left(w_{1}, w_{2}\right)^{\prime}= & \left(\frac{1}{3} z^{3}-3 z-32 / 3,-z^{2}+4 / 3\right)-(3 z+8)\left(w_{1}, w_{2}\right) \\
& -\left(w_{1}, w_{2}\right)\left(\begin{array}{cc}
-\frac{1}{3} z^{3}+z+32 / 3 & z^{2}-4 / 3 \\
-\frac{2}{3} z^{3}+6 z+9 / 3 & 2 z^{2}-2 z-8 / 3
\end{array}\right) \\
& --\left(w_{1}, w_{2}\right)\left(\begin{array}{c}
z+8 \\
2 z+19
\end{array}\right)\left(w_{1}, w_{2}\right) .
\end{aligned}
$$

This gives the system

$$
\left(\begin{array}{cc}
u_{11} & u_{12} \\
u_{21} & u_{22} \\
v_{1} & v_{2}
\end{array}\right)^{\prime}=\left(\begin{array}{ccc}
-\frac{1}{3} z^{3}+z+32 / 3 & z^{2}-4 / 3 & z+8 \\
-\frac{2}{3} z^{3}+6 z+9 / 3 & 2 z^{2}-2 z-8 / 3 & 2 z+19 \\
\frac{1}{2} z^{3}-3 z-32 / 3 & -z^{2}+4 / 3 & 3 z+8
\end{array}\right)\left(\begin{array}{cc}
u_{11} & u_{12} \\
u_{21} & u_{22} \\
v_{1} & v_{2}
\end{array}\right)
$$


with the solutions

$$
W=\left(w_{1}, w_{2}\right)=\left(v_{1}, v_{2}\right)\left(\begin{array}{ll}
u_{11} & u_{12} \\
u_{21} & u_{22}
\end{array}\right)^{-1}
$$

In the formal fundamental system we have from ELISE

$$
\exp Q\left(z^{1 / p}=\exp \left(\left(\begin{array}{ccc}
-\frac{1}{12} z^{4}+\frac{2}{3} z^{3} & 0 & 0 \\
0 & -z^{2} & 0 \\
0 & 0 & -z^{2}
\end{array}\right)\right),\right.
$$

and the 12 Stokes directions from $M(Q)$ are given by $\gamma_{1}=\pi / 8, \gamma_{2}=\pi / 4$, $\gamma_{3}=3 \pi / 8(\bmod \pi / 2)$. The eigenvalues of the matrix $L$ in $(2.1)$ are zero (mod an integer). The CPU-time for ELISE was 17 seconds.

Since we have only real eigenvalues, from the remark at the end of Section 3 and (3.31), for any solution we have at most a finite number of poles outside the $\Lambda$-domains around the Stokes directions. Notice now that $M(Q)$ and $M_{1}(Q)$ here define the same set of Stokes directions; hence the critical points of any solution are-except for a finite number-in the same regions as the poles. Furthermore, neither in Theorem 4.1 nor in Theorem 4.2 the unpleasant domains $B_{k}(\delta)$ appear in this example.

\section{Acknowledgements}

I wish to thank the referees and F. Brüggemann for their helpful remarks and discussions.

\section{References}

[1] L. V. Ahlfors, Complex analysis, [3rd edition], (McGraw Hill, New York-TorontoLondon 1979).

[2] W. Balser, "Einige beiträge zur invariantentheorie meromorpher differential-gleichungen", Habil-Schrift, Ulm 1978.

[3] V. Dietrich, "Newton-Puiseux-Diagramm für systeme linearer differentialgleichungen", Complex Variables Theory Appl. 7 (1987), 265-296.

[4] V. Dietrich, "ELISE, an algorithm to compute asymptotic representations, realized with the computer algebra system MAPLE", preprint, 1989.

[5] V. Dietrich, "Über die annahme der möglichen wachstumsordnungen and typen bei linearen differentialgleichungen, Habiltationsschrift", RWTH Aachen, 1990.

[6] P. Henrici, Applied and computational complex analysis, Vol. II, (John Wiley, New York-London-Sydney-Toronto, 1977).

[7] G. A. Hewer, "Periodicity, detectability and the Matrix Riccati equation", SIAM J. Control 13 (1975) 1235-1251. 
[8] G. Jank and L. Volkmann, Meromorphe funktionen und differentialgleichungen, UTBGroße Reihe, Birkhäuser Verlag, Basel-Boston-Stuttgart, 1985.

[9] G. Jank, "Growth properties and values distribution of transcendental solutions of Riccati differential equations", preprint, 1989.

[10] W. B. Jurkat, Meromorphe differentialgleichungen, Lecture Notes in Mathematics No. 637, (Springer Verlag, Berlin-Heidelberg-New York, 1978).

[11] R. B. Leipnik, "A canonical form and solution for the Matrix Riccati Differential Equation", J. Austral Math. Soc. Ser. B, 26 (1985) 355-361.

[12] B. J. Lewin, Nullstellenverteilung ganzer funktionen, (Akademie Verlag, Berlin, 1962).

[13] R. Nevanlinna, "Zur Theorie der meromorphen Funktionen", Acta Math., 46 (1925) 1-99.

[14] W. T. Reid, Riccati differential equations, (Academic Press, New York-London, 1972).

[15] W. Wasow, Asymptotic expansions for ordinary differential equations, (John Wiley, New York, 1965). 